\title{
An Audit of the Emergency Airway Service in a Regional Hospital in Singapore
}

\author{
Sheng Chuu Anne Kiew*, Hui Xin Marilyn Ng, Kelvin How Yow Quek, Jinxi Zheng, Joanne Yeo \\ Department of Anaesthesiology and Surgical Intensive Care, Changi General Hospital, Singapore \\ Email: *anne.kiew.s.c@singhealth.com.sg
}

How to cite this paper: Kiew, S.C.A., Ng, H.X.M., Quek, K.H.Y., Zheng, J.X. and Yeo, J. (2021) An Audit of the Emergency Airway Service in a Regional Hospital in Singapore. Open Journal of Anesthesiology, 11, 195-206.

https://doi.org/10.4236/ojanes.2021.117019

Received: June 3, 2021

Accepted: July 6, 2021

Published: July 9, 2021

Copyright $\odot 2021$ by author(s) and Scientific Research Publishing Inc. This work is licensed under the Creative Commons Attribution International License (CC BY 4.0).

http://creativecommons.org/licenses/by/4.0/

\begin{abstract}
Background: Emergency endotracheal intubations (EEI) performed outside of operating theatre (OT) tend to be more challenging and associated with higher risk of complications. In 2011, with the objective of improving patient outcomes, we set up an Emergency Airway Service (EAS) at our 1000-bed regional hospital, with the aim of providing specialized assistance for outside of OT difficult airway management. Method: A retrospective audit of EAS activation from 12/9/2016 and 27/10/2020 was conducted. EAS forms and electronic medical records were reviewed. We collected information on patient characteristics, EAS activation characteristics and its outcomes. Descriptive analysis method was used to present the collected data. Results: There were a total of 275 activations, of which 268 were analysed. Reasons for activation were anticipated difficult intubation $(42.2 \% \mathrm{n}=113)$, failed intubation attempt $(52.6 \%, \mathrm{n}=141)$ and advanced intubation equipment required $(5.2 \%$ $\mathrm{n}=14)$. Intubation was attempted in $261 / 268(97.4 \%)$ cases by the EAS team. Of these, 255 (97.7\%) cases were successful while $6(2.3 \%)$ cases failed intubation. Of the successful intubations by the EAS team, 208/255 (81.5\%) were successful on the first attempt. Out of the 6 unsuccessful intubation cases, 1 case required a rescue cricothyroidotomy and 4 cases required an open tracheostomy. Intubation was deemed easy by the EAS team in 170/261 (65.1\%) cases. 64/170 (37.6\%) cases were intubated with a video laryngoscope (VL). There were 85 cases $(32.3 \%)$ classified as difficult intubation by the EAS specialist, 13/85 (15.3\%) were intubated using only VL, 54/85 (63.5\%) cases were intubated using VL with style/bougie. Conclusion: Audit results showed that providing an experienced and well-equipped team of airway specialists round-the-clock to assist in difficult and potentially difficult endotracheal intubations is justifiable and may reduce complications associated with EEI.
\end{abstract}

\section{Keywords}

Difficult Airway, Emergency Endotracheal Intubation, 
Airway Response Team, Airway Service

\section{Introduction}

Emergency endotracheal intubation (EEI) is a critical life-saving intervention performed both inside and outside the operating theatre (OT). Compared to elective intubations, emergency intubations tend to be more challenging and chaotic [1] [2]. This is usually due to a combination of factors that lead emergency intubations to be potentially fraught with risk. The operator is frequently presented with a rapidly deteriorating situation or a patient in extremis, with minimal to no time to perform a thorough airway assessment prior to intubation. In addition, patients may be haemodynamically compromised and are rarely fasted. Furthermore, out of OT intubations often take place in remote locations with limited access to advanced airway management equipment, and may be performed by operators who have little to no training in airway management. One study report noted a $10.3 \%$ incidence of difficult intubation in intubation carried out outside the OT in a university hospital setting [3].

Whilst complication rates of elective intubations performed in the OT complex are low [4], the risk of complications such as failed intubation, hypoxia, hypotension, aspiration and airway trauma are much higher when performed in urgent conditions outside of the OT, and may exceed 20\% [1] [5] [6] [7]. Even within the OT complex in ideal situations, endotracheal intubation performed by inexperienced operators is associated with higher risks [7] [8], and being out of OT where the first responders may have no practical experience with airway management exacerbates these risks. Not surprisingly, studies have shown that emergent airway management procedures performed by senior anaesthesia residents as first responders have a lower incidence of complications [2] [6].

In 2011, with the objective of improving patient outcomes, we set up an Emergency Airway Service (EAS) at our 1000-bed regional hospital. The EAS aims to provide round-the-clock, specialised assistance for difficult airway management and comprises an anaesthesia specialist and a trained anaesthetic nurse. Airway adjuncts and advanced airway management equipment such as a video laryngoscope (VL), a fibreoptic bronchoscope (FOB) and equipment for surgical airway access are immediately available in an accompanying grab bag and trolley. Equipment that our staff was familiar with, in accordance with the recommendations by the Difficult Airway Society guidelines, and that could be stored in the trolley was chosen [9]. The team is activated via a dedicated emergency phone number to the hospital telephone operator which activates the team via an allocated airway telephone. The team can be activated by any responder to an emergent patient situation but does not need to be activated for all out of OT airway interventions. Prior to the EAS, activation of skilled anaesthetic assistance caused greater delay (as the number for the anaesthesia specialist had to be sourced), and out-of-OT equipment was not readily available. The decision to 
activate may have been delayed, potentially leading to unfavourable outcomes. This scenario was similar to that at John Hopkins Hospital and Grant Medical Center in the lead-up to the commencement of their airway response teams [8] [10].

In this audit, we aim to evaluate the utilisation and efficacy of this service.

\section{Methods}

An airway management team was set up to provide assistance in emergency endotracheal intubations performed in all locations outside the OT.

An EAS record form was completed post-activation by the anaesthesia specialist involved.

We conducted a retrospective audit of EAS activation forms between 12/9/2016 and 27/10/2020. Data from EAS commencement in 2011 to 11/9/2016 was not included in the audit as prior to $12 / 9 / 2016$, there was no standardised documentation of the activations.

Information reviewed included reason for intubation, the patient characteristics that led to EAS activation, any details on attempts prior to EAS activation, the assistance provided by the EAS team (airway assessment, successful techniques use, number of attempts) and outcomes. The rate of successful rescue of failed intubation was used as a marker of efficacy of EAS.

As all data was anonymised, we obtained an exemption from review by the Singhealth Centralised Institutional Review Board. Descriptive analysis was used to present the collected data.

\section{Results}

There were a total of 275 activations between 12/9/2016 and 27/10/2020, of which 268 were analysed in our audit. The remaining 7 activation were excluded from the audit as the EAS record forms were incompletely filled.

\subsection{Patient Characteristics}

The patient characteristics and factors leading to activation of EAS for endotracheal intubation are summarised in Table 1.

Reasons for endotracheal intubations were respiratory failure, cardiac arrest, airway pathology and altered mental status (Figure 1).

\subsection{Activation Characteristics}

The locations of EAS activations were the General Ward (26.4\%, $\mathrm{n}=71$ ), Accident and Emergency (A\&E) $(19.4 \%, \mathrm{n}=52)$, Medical Intensive Care Unit (MICU) $(24.6 \%, \mathrm{n}=66)$, High Dependency $(14.1 \%, \mathrm{n}=38)$, Surgical Intensive Care Unit $(0.37 \%, \mathrm{n}=1)$, other locations $(6 \% \mathrm{n}=16)$ (e.g. endoscopic center, cardiac catheterization laboratory, radiology department) and missing data (8.9\%, $\mathrm{n}=24)$.

Reasons for activation were anticipated difficult intubation $(42.2 \% \mathrm{n}=113)$, 
Table 1. Patient characteristics.

\begin{tabular}{cc}
\hline Patient Characteristics & $\mathrm{N}=268(\%)$ \\
\hline Gender: & $159(59.3 \%)$ \\
Male & $109(40.6 \%)$ \\
Female & $128(47.7 \%)$ \\
Obese (BMI $\geq 35)$ & $84(31.3 \%)$ \\
Short Thyromental Distance $(<6 \mathrm{~cm})$ & $19(7.1 \%)$ \\
Restricted Mouth Opening & $43(16 \%)$ \\
Reduced Neck mobility & $23(8.5 \%)$ \\
Bad Dentition & $23(8.5 \%)$ \\
Prev surgery/Radiotherapy & $13(4.9 \%)$ \\
Airway lesion & $72(26.9 \%)$ \\
Blood/secretions &
\end{tabular}

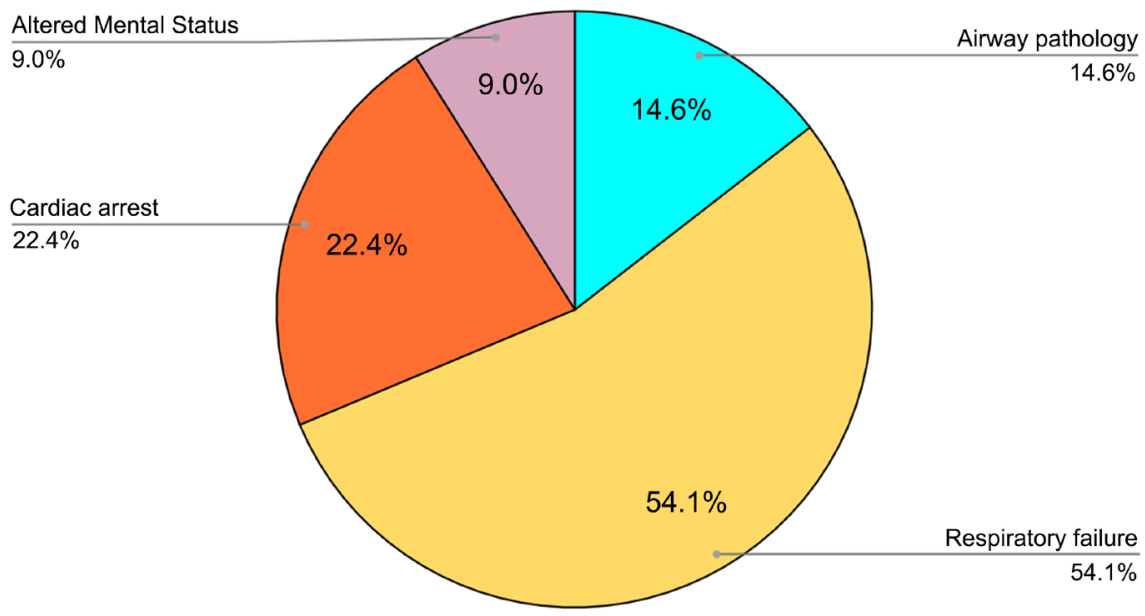

Figure 1. Reason for endotracheal intubation.

failed intubation attempt $(52.6 \%, \mathrm{n}=141)$ and advanced intubation equipment required $(5.2 \%, \mathrm{n}=14)$. Of note, there was a similar likelihood of the team being activated for an anticipated difficult airway, as for a failed intubation attempt. In the second situation, the experience of the first responder varied from junior inexperienced medical staff to emergency or intensive care specialists.

\subsection{Activation Outcomes}

Intubation was attempted in $261 / 268(97.4 \%)$ cases by the EAS team. Of these, $255(97.7 \%)$ cases were successful while $6(2.3 \%)$ cases failed intubation.

Of the successful intubations by the EAS team, 208/255 (81.5\%) were successful on the first attempt.

Out of the 6 unsuccessful intubation cases, 1 case required a rescue cricothyroidotomy and 4 cases required an open tracheostomy. Endotracheal intubation was abandoned in the remaining case as cardiopulmonary resuscitation was deemed futile and resuscitative efforts were terminated. 
In the seven cases where intubation was not attempted, three cases were already intubated by the time the EAS team arrived and in two cases the patient's condition improved and intubation was not required.

Intubation was deemed easy by the EAS team in 170/261 (65.1\%) cases. 64/170 (37.6\%) cases were intubated with a video laryngoscope (VL), and, 84 cases (49.4\%) used VL together with a stylet or a bougie. Only 8 cases were intubated using direct laryngoscope (DL) and 9 cases using DL with stylet/bougie. 3 cases used fiberoptic bronchoscope (FOB) and 2 cases used FOB with VL (Figure 2).

There were 85 cases $(32.3 \%)$ classified as difficult intubation by the EAS specialist. 13/85 (15.3\%) cases were intubated using only VL, 54/85 (63.5\%) cases required VL with stylet/bougie, and 10/85 (11.8\%) were intubated with a FOB. Only 5/85 (5.9\%) cases were successfully intubated using DL with stylet/bougie and 3/85 (3.5\%) cases used VL + FOB (Figure 3).

6 cases were deemed impossible to intubate. 5 out of the 6 cases required a surgical airway and in 1 case intubation was abandoned as further resuscitation was deemed futile.

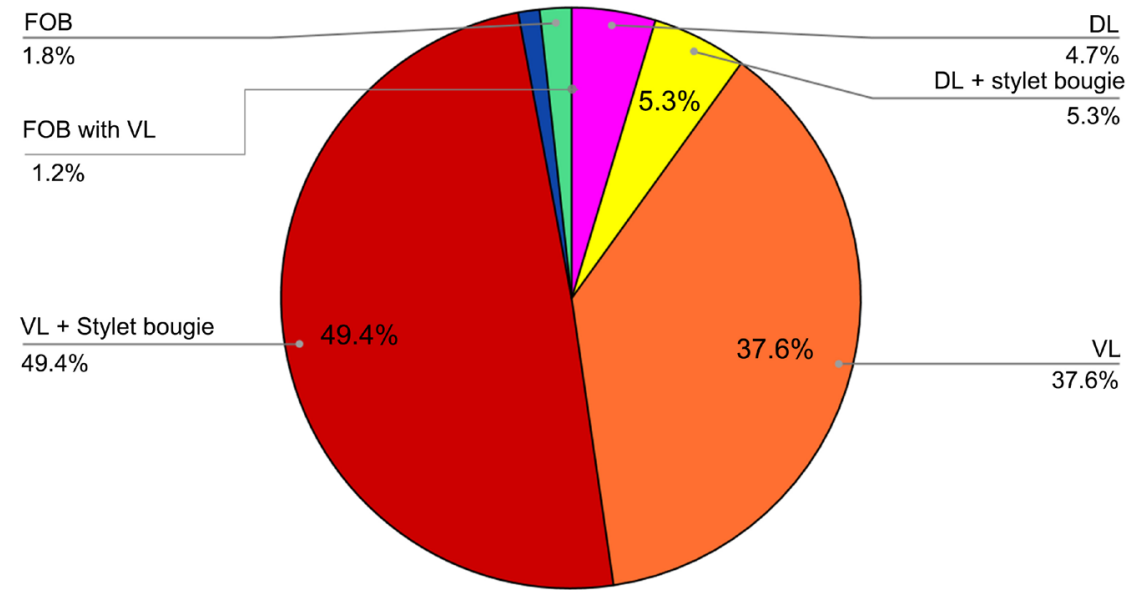

Figure 2. Intubation technique in easy intubation group.

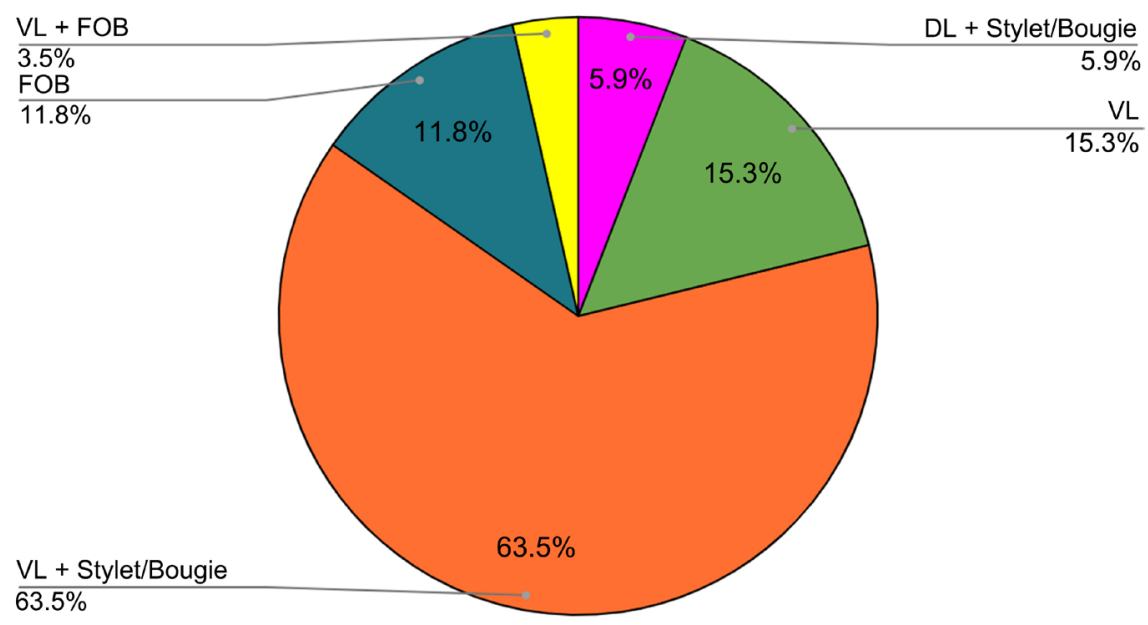

Figure 3. Intubation technique in difficult intubation group. 
No muscle relaxant was used by the primary team prior to EAS activation in $116 / 268$ (43.2\%) cases. 120/268 (44.8\%) cases were given suxamethonium, 15/268 (5.6\%) cases were given rocuronium and 12/268 (4.5\%) cases were given atracurium. No data on muscle relaxant was recorded in 5 cases.

\section{Discussion}

Patients requiring emergency endotracheal intubation are frequently unfasted, rapidly deteriorating and critically ill. The importance of securing an emergency airway as quickly and efficiently as possible cannot be overstated. Repeated intubation attempts are associated with a greatly increased risk of complications like airway oedema, hypoxia, regurgitation of gastric content, airway mucosal trauma and oesophageal intubation [1] [7] [11]. Multiple attempts may also convert what was an easy airway into a difficult one due to the trauma and oedema. We report an over $80 \%$ successful first-pass intubation rate by our EAS team, in a population where $42 \%$ of patients were identified as potentially difficult by the first-responder team, and $52 \%$ had already failed at least one intubation attempt.

It is clear from our data that the VL is the rescue device of choice by the EAS team. Of the patients deemed easy intubation by our team, $87 \%$ were successful with a VL, or VL with a stylet or bougie, while of those considered difficult (32.3\% of activations), $78.8 \%$ were eventually successful using either VL only or VL combined with either a stylet or a bougie. There is good evidence that the VL not only improves the glottic view, but also shortens intubation time, and improves first pass success rates [11] [12] [13] [14]. A Cochrane review of 64 RCTs suggested that the VL is associated with fewer failed intubations in both predicted difficult and all laryngoscopy, more Grade 1 views, greater ease of use and less airway trauma [15]. Correspondingly, the National Emergency Airway Registry (NEAR) in the United States has noted an increased rate of first pass intubation rate as the percentage use of VL has increased over the years [16] [17]. As such, we suggest that the VL be used as the first choice device during emergency tracheal intubation.

In addition, when the service was introduced, it was decided to provide a range of airway equipment beyond the basic equipment available in remote locations. This gradually evolved during our service based on feedback from the practitioners involved. Initially a grab bag with a $\mathrm{McGrath}^{\mathrm{TM}}$ videoscope and a small amount of extra equipment was provided. However, review after commencement of the service revealed that a more extensive set of equipment would be useful, so a portable trolley with greater range of equipment, almost equivalent to that of a difficult airway trolley in the operating complex was provided. This is pushed to the activating location by a trained anaesthetic assistant. The trolley (Figure 4) contains a VL (the $\mathrm{CMAC}^{\circledR}$ with a variety of blades including the D blade), a $\mathrm{CMAC}^{\circledR}$ bronchoscope, adjuncts such as oropharyngeal and nasopharyngeal airways in various sizes and airway rescue equipment such as laryngeal masks and equipment for emergency cricothyroidectomy. In addition 


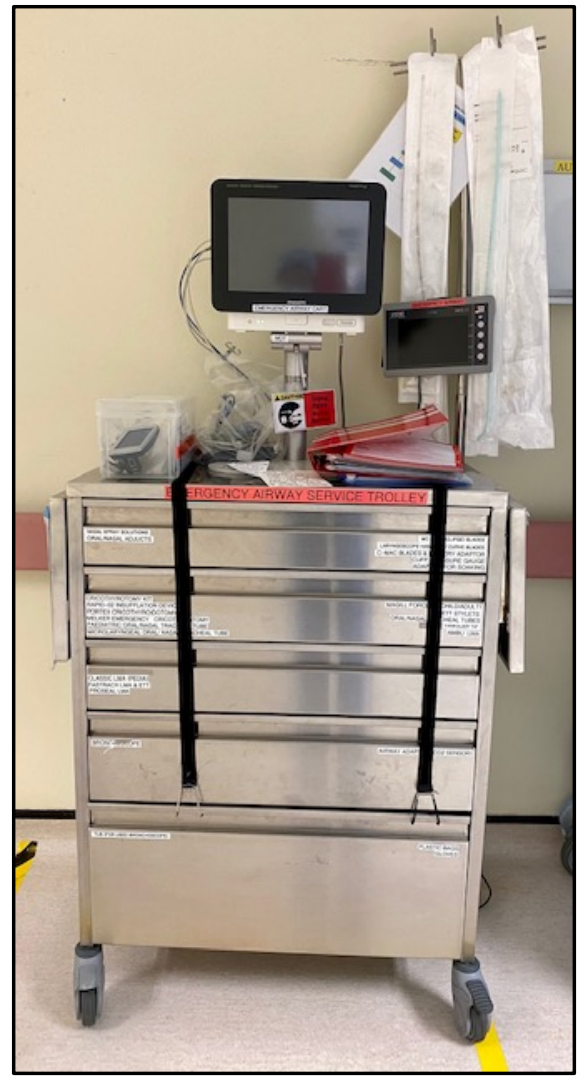

Figure 4. Emergency airway trolley.

the grab bag remains readily available as it is more portable and allows the specialist to commence management if the assistant with the trolley is delayed. The range of equipment in the bag and trolley is regularly re-evaluated to ascertain which are most used and is standardized to the equipment in our OT difficult airway trolleys, which anaesthesia specialists in the hospital are familiar from OT and simulation training. Finally, all anaesthesia specialists in our institution undergo yearly refresher sessions on how to perform an emergency cricothyroidectomy with the equipment available in the airway trolley.

It must also be highlighted that the presence of an experienced assistant familiar with difficult airway management, in addition to the equipment available in the grab bag and trolley, is invaluable in an emergency. Activation of the EAS mobilises both an anaesthetic specialist and an experienced anaesthetic unit nurse as the skilled assistant.

Previous studies have shown that a higher first pass emergency intubation rate is also associated with increased operator clinical experience [6] [11] [18]. As we can see from our data, the majority of EAS activation was from General Ward (26.4\%) followed by the medical ICU (24.6\%) and A\&E (19.4\%). The majority of physicians working in the general ward are likely to have little experience in airway management, and they are therefore more likely to require assistance. This is perhaps reflected by the low rate of use of muscle relaxants in intubation attempts by the primary responders as these drugs are perhaps perceived to in- 
terfere with the return to spontaneous respiration should intubation fail. Although our retrospective analysis cannot ascertain the reasons for failed initial intubation attempt, we theorize that on top of the lack of airway experience, the failure to use muscle relaxants would have contributed to the difficulty of intubation in many of these cases. Several randomised trials and observational studies have shown that the use of neuromuscular blocking agents during emergency tracheal intubation improves success rates while reducing complications [19] [20]. In fact, in 2018 a meta-analysis on the avoidance of the use of muscle relaxants during tracheal intubation showed that intubation was considered difficult in $56.3 \%$ of patients when neuromuscular blocking agents were not used, and this was reduced to $4.7 \%$ when they were used [21]. Hence we feel that in the absence of contraindications, and in experienced hands, neuromuscular blocking agents should be used in emergency tracheal intubation.

From our data, it is interesting to note that more than $40 \%$ of EAS activations were for anticipated difficult airways. The credit for this must go to improved education efforts by the various departments in our institution. In addition, easy activation (a single call to the hospital operator from any line in the hospital) and publicity for the service since its introduction in 2011 has also helped.

There are several limitations to this audit. Firstly, this is a retrospective audit and hence it is subject to incomplete or missing data. This is also a single-center audit, and the workflow and results may not be generalisable to all health institutions. Generalisation of the service to other health institutions will be somewhat limited by variations in staffing, equipment, costs and operational considerations. However, the data from our centre and at least two other centres with similar emergency airway response teams [8] [22] have all shown positive outcomes for the patients who were managed by a dedicated difficult airway team. In comparison to John Hopkin's and Grant Medical Centre's emergency airway teams [8] [21], which included the surgeons, particularly head and neck or otolaryngology (ENT), emergency specialists and a range of other staff such as respiratory specialists, our hospital's EAS team has been solely staffed by anaesthetic specialists with the support of skilled anaesthetic nurses. This may reflect regional variations in practice, as our institution does not have 24 -hour on site specialist ENT support.

Furthermore, as data was not collected prior to the commencement of the service or with initial phases of the service, our evidence of benefit beyond anecdotal evidence is limited. However worldwide, difficult airway societies have made recommendations for the use of such emergency airway services and other institutions such as John Hopkins and Grant Medical Center created their teams directly in response to serious airway events, and have demonstrated no patient morbidity or mortality from the activations of their teams [8] [23]. Going forward, a well-designed study to evaluate the effectiveness of the service in reducing patient morbidity and mortality related to EEI, reduction in length of hospital stay and decreasing cost of patient care would be ideal.

There is currently no specific section on our form documenting the reasons 
for failed intubation by the primary responders or the complications of EEI, so we are unable to ascertain the factors contributing to failed intubation by primary responders or complication rates of EEI. We suggest that the EAS form should be revised in the future to include information such as the reasons for failed intubation by first responders, complications related to EEI, activation to intubation time, length of hospital stay and 30 days mortality, to better enable us to monitor the effectiveness of the service. In future, the EAS form should be migrated to an electronic database to allow better data capture and minimize missing data. This would also assist in improving the service by easier monitoring of our activation to assistance time as well as ensuring the consistency of the service across all providers and monitoring for complications. Currently, our service does not monitor for morbidity and mortality related to the activation, but this is important to allow identification of deficiencies in the current set-up and if any further improvement is needed.

The data on the total number of EEI performed within the institution over the period of time was not available, making it impossible to ascertain the incidence of EAS activation and missed activations. Data on adverse outcomes of all EEI also not available and hence we were unable to determine the effectiveness of EAS in reduction of EEI complications. A cross disciplinary approach at a senior level would allow us to gather data on total number of EEI performed and identify missed activations and if we needed to better implement airway assessment teaching to ward staff along the lines of John Hopkins and Grant Medical Center [8] [21].

Audit of the airway service should also be conducted at regular interval to identify measures to enhance the implementation of the service. This is in keeping with the NAP4 guidelines [24]. As the current trolley is bulky, usage of equipment should be reviewed at regular intervals and a standardised guideline for review could be implemented rather than ad hoc discussion between the department specialists. Equipment deemed underutilised can then be removed in a more uniform fashion, with input from the anaesthetic department. This may make the trolley more compact and portable and would also make space for any future new equipment additions. There are of course exceptions, such as the surgical airway equipment. The equipment is regularly checked for expiry and replaced.

With the current Covid-19 pandemic, we have also added two sets of powered air purifying respirators for the safety of our staff. In addition, Covid-19 positive patients (or likely-infected patients pending swab results) now warrant EAS activation within our institution to minimize aerosol exposure to staff, with the aim of achieving a single successful intubation attempt by an experienced operator. It is as yet unclear how much this has altered the number of activations for our team and the benefit of this measure should be assessed in future audits.

\section{Conclusion}

The Emergency Airway Service in our institution has successfully assisted in more 
than 250 emergency endotracheal intubations to date. Our results show that providing an experienced and well-equipped team of airway specialists round-the-clock to assist in difficult and potentially difficult endotracheal intubations is justifiable and may potentially help to reduce complications associated with EEI. Modifications to our data collection form will allow us to better ascertain the clinical and outcome variables and guide us in implementing any required changes.

\section{Conflicts of Interest}

The authors declare no conflicts of interest regarding the publication of this paper.

\section{References}

[1] Mort, T.C. (2004) Emergency Tracheal Intubation: Complications Associated with Repeated Laryngoscopic Attempts. Anesthesia \& Analgesia, 99, 607-613. https://doi.org/10.1213/01.ANE.0000122825.04923.15

[2] Schmidt, U.H., Kumwilaisak, K., Bittner, E., George, E. and Hess, D. (2008) Effects of Supervision by Attending Anesthesiologists on Complications of Emergency Tracheal Intubation. Anesthesiology, 109, 973-977. https://doi.org/10.1097/ALN.0b013e31818ddb90

[3] Sollid, S.J.M., Mellin-Olsen, J. and Wisborg, T. (2016) Emergency Airway Management-By Whom and How? Acta Anaesthesiologica Scandinavica, 60, 1185-1187. https://doi.org/10.1111/aas.12759

[4] Domino, K.B., Posner, K.L., Caplan, R.A. and Cheney, F.W. (1999) Airway Injury during Anesthesia: A Closed Claims Analysis. Anesthesiology, 91, 1703-1711. https://doi.org/10.1097/00000542-199912000-00023

[5] Schwartz, D.E., Matthay, M.A. and Cohen, N.H. (1995) Death and Other Complications of Emergency Airway Management in Critically Ill Adults: A Prospective Investigation of 297 Tracheal Intubations. Anesthesiology, 82, 367-376. https://doi.org/10.1097/00000542-199502000-00007

[6] Martin, L.D., Mhyre, J.M., Shanks, A.M., Tremper, K.K. and Kheterpal, S. (2011) 3,423 Emergency Tracheal Intubations at a University Hospital: Airway Outcomes and Complications. Anesthesiology, 114, 42-48.

https://doi.org/10.1097/ALN.0b013e318201c415

[7] Cham, E., Wong, O. and Yip, K. (2016) Clinical Practice and Risk Factors for Immediate Complications of Endotracheal Intubation by Intensive Care Unit Doctors in a Regional Hospital in Hong Kong. Hong Kong Journal of Emergency Medicine, 23, 135-144. https://doi.org/10.1177/102490791602300302

[8] Mark, L.J., Herzer, K.R., Cover, R., Pandian, V., Bhatti, N.I., Berkow, L.C., Haut, E.R., Hillel, A.T., Miller, C.R., Feller-Kopman, D.J., Schiavi, A.J., Xie, Y.J., Lim, C., Holzmueller, C., Ahmad, M., Thomas, P., Flint, P.W. and Mirski, M.A. (2015) Difficult Airway Response Team: A Novel Quality Improvement Program for Managing Hospital-Wide Airway Emergencies. Anesthesia \& Analgesia, 121, 127-139. https://doi.org/10.1213/ANE.0000000000000691

[9] (2015) DAS Guidelines for Management of Unanticipated Difficult Intubation in Adults. https://das.uk.com/files/das2015intubation_guidelines.pdf

[10] Chmielewska, M., Winters, B.D., Pandian, V. and Hillel, A.T. (2015) Integration of a Difficult Airway Response Team into a Hospital Emergency Response System. 
Anesthesiology Clinics, 33, 369-379. https://doi.org/10.1016/j.anclin.2015.02.008

[11] Jung, W. and Kim, J. (2020) Factors Associated with First-Pass Success of Emergency Endotracheal Intubation. The American Journal of Emergency Medicine, 38 , 109-113. https://doi.org/10.1016/j.ajem.2019.09.001

[12] Sakles, J.C., Mosier, J.M., Patanwala, A.E., Dicken, J.M., Kalin, L. and Javedani, P.P. (2015) The C-Mac Video Laryngoscope is Superior to the Direct Laryngoscope for the Rescue of Failed First Attempt Intubations in the Emergency Department. Journal of Emergency Medicine, 48, 280-286. https://doi.org/10.1016/j.jemermed.2014.10.007

[13] Vassiliadis, J., Tzannes, A., Hitos, K., Brimble, J. and Fogg, T. (2015) Comparison of the C-MAC Video Laryngoscope with Direct Macintosh Laryngoscopy in the Emergency Department. Emergency Medicine Australasia, 27, 119-125. https://doi.org/10.1111/1742-6723.12358

[14] Arulkumaran, N., Lowe, J., Ions, R., Mendoza, M., Bennett, V. and Dunser, M.W. (2018) Videolaryngoscopy Versus Direct Laryngoscopy for Emergency Orotracheal Intubation Outside the Operating Room: A Systematic Review and Meta Analysis. British Journal of Anaesthesia, 120, 712-724. https://doi.org/10.1016/j.bja.2017.12.041

[15] Lewis, S.R., Butler, A.R., Parker, J., Cook, T.M. and Smith, A.F. (2016) Videolaryngoscopy Versus Direct Laryngoscopy for Adult Patients Requiring Tracheal Intubation. Cochrane Database of Systematic Reviews, 11, Article No. CD011136. https://doi.org/10.1002/14651858.CD011136.pub2

[16] Cook, T.M. (2018) Strategies for the Prevention of Airway Complications-A Narrative Review. Anaesthesia, 73, 93-111. https://doi.org/10.1111/anae.14123

[17] Brown, C.A., Bair, A.E., Pallin, D.J., Laurin, E.G., Walls, R.M. and National Emergency Airway Registry (NEAR) Investigators. (2010) Improved Glottic Exposure with the Video Macintosh Laryngoscope in Adult Emergency Department Tracheal Intubations. Annals of Emergency Medicine, 56, 83-88.

https://doi.org/10.1016/j.annemergmed.2010.01.033

[18] Sagarin, M.J., Barton, E.D., Chng, Y.-M., Walls, R.M. and On Behalf of the National Emergency Airway Registry (NEAR) Investigators. (2005) Airway Management by US and Canadian Emergency Medicine Residents: A Multicenter Analysis of More Than 6,000 Endotracheal Intubation Attempts. Annals of Emergency Medicine, 46, 328-336. https://doi.org/10.1016/j.annemergmed.2005.01.009

[19] Li, J., Murphy-Lavoie, H., Bugas, C., Martinez, J. and Preston, C. (1999) Complications of Emergency Intubation with and without Paralysis. The American Journal of Emergency Medicine, 17, 141-143. https://doi.org/10.1016/S0735-6757(99)90046-3

[20] Wilcox, S.R., Bittner, E.A., Elmer, J., Seigel, T.A., Nguyen, N.T., Dhillon, A., Eikermann, M., Schmidt, U. (2012) Neuromuscular Blocking Agent Administration for Emergent Tracheal Intubation is Associated with Decreased Prevalence of ProcedureRelated Complications. Critical Care Medicine, 40, 1808-1811. https://doi.org/10.1097/CCM.0b013e31824e0e67

[21] Lundstrøm, L.H., Duez, C.H.V., Nørskov, A.K., Rosenstock, C.V., Thomsen, J.L., Moller, A.M., Strande, S. and Wetterslev, J. (2018) Effects of Avoidance or Use of Neuromuscular Blocking Agents on Outcomes in Tracheal Intubation: A Cochrane Systematic Review. British Journal of Anaesthesia, 120, 1381-1393. https://doi.org/10.1016/j.bja.2017.11.106

[22] Tan, H., Lew, T. and Earnest, A. (3002) Emergency Airway Service by Anaesthetists: A Selective Referral Approach. Critical Care, 6, Article No. P248. 
https://doi.org/10.1186/cc1716

[23] Long, L., Vanderhoff, B., Smyke, N., Shaffer, L.E., Solomon, J. and Steuer, J.D. (2010) Management of Difficult Airways Using a Hospital-Wide "Alpha Team" Approach. American Journal of Medical Quality, 25, 297-304. https://doi.org/10.1177/1062860610366587

[24] Cook, T.M., Woodall, N., Harper, J. and Benger, J. (2011) Major Complications of Airway Management in the UK: Results of the 4th National Audit Project of the Royal College of Anaesthetists and the Difficult Airway Society. Part 2: Intensive Care and Emergency Departments. British Journal of Anaesthesia, 106, 632-642. https://doi.org/10.1093/bja/aer059 